\title{
Quality in Healthcare: An Innovative Adaptable System for In-patient Rooms
}

\author{
Michele Di Sivo ${ }^{1}$, Claudia Balducci ${ }^{2}$ \\ ${ }^{1}$ Department of Engineering of Energy, Systems, Territory and Construction, University of Pisa, Pisa, Italy \\ ${ }^{2}$ Department of Architecture, G. D'Annunzio University of Chieti- Pescara, Pescara, Italy
}

Email address:

michele.disivo@unipi.it (M. Di Sivo)

\section{To cite this article:}

Michele Di Sivo, Claudia Balducci. Quality in Healthcare: An Innovative Adaptable System for In-patient Rooms. International Journal of Architecture, Arts and Applications. Vol. 5, No. 1, 2019, pp. 10-17. doi: 10.11648/j.ijaaa.20190501.12

Received: December 3, 2018; Accepted: December 28, 2018; Published: March 26, 2018

\begin{abstract}
This article aims to study methodologically hospital buildings and, in particular, the design of inpatient rooms, identified as the place of maximum convergence between the renewed framework of the hospital and its users. The definition of design guidelines to improve and qualify the in-patient rooms, enhancing their performance, takes into account the fact that the established requirements of medical protocols must be accompanied by those new requirements related to the massive evolution in health organizations. Changes in medicine, culture and society have necessarily led to a new formulation of the hospital model and, over the last few decades, the massive introduction of technology within the hospital has conditioned the morphological-space configuration and the presence of new relationships and spatial functions in the hospitals. The research of this article attempts, therefore, to explore the use of technology to improve the quality of patient rooms' design, formulating a model that can adapt to economic, social, health and technological changes, and, at the same time, can ensure that the system, services and activities meet the evolving needs of the population.
\end{abstract}

Keywords: Patient Rooms, Patient-Centeredness, Acuity Adaptable, Technology, Healthcare Structures, Strategies

\section{Introduction}

The rapid and constant development in medical and scientific fields requires continuous updating of the typological models and a continuous transformation of the hospital buildings. However, one of the most critical aspects to consider is the process of adapting structures to such rapid evolution.

The health sector, in fact, is rapidly changing its nature and the origin of these transformations is due to the progress of research in the medical field and even more to an ever-increasing amount of biomedical equipment and advanced technologies for diagnosis and therapies and healthcare facilities [1].

Due to a process of miniaturization of the components and to the possibility of easy transfer of some medical technologies, such as X-ray equipment, it is possible to bring these technologies directly to the patient's bed, creating a real Bedside Point of Care. It is a model of care based on the patient-centeredness which reduces the need to transfer the patient, making each room a focal point of the hospital.

The development and adapting of smaller and more mobile technologies have consequently led to an increase in the hospital room area and a substantial rethinking of its configuration. This reconfiguration takes into account new activities, users and relationships, and also makes it possible to bring almost all the activities and equipment that concern the patient and that are functional to his healing, closer to the bed. So, rather than defining the size of the room, it is crucial to quantify the space necessary for the activities that take place around the patient's bed, for example the movement of life support systems. For this reason, international guidelines have been created that establish a minimum space around the bed instead of referring to the overall area of the room. This minimum space is called the Ergonomic Envelope [2] by the Anglo-Saxons and Minimum Clearance by the Americans - "clearances for the bed in $\mathrm{med} / \mathrm{surg}$ rooms are 36 inches, 48 inches on the transfer side, and 36 inches at the foot of the bed." [3].

This approach (the bedside point of care) is equitable to the Acuity Adaptable system, a system that adapts to level of care needed and has introduced an innovative model. This model has led to measurable benefits for the well-being and the healing process of the patients [4]. "The acuity-adaptable care delivery" is a model adopted in United States hospitals, 
in order to promote adaptability to frequent changes in the needs of the hospitals, and to ensure a continuous flow of patients within the facility. Many research [5-10], based on empirical studies, attest to numerous positive effects, benefits and opportunities arising from the use of Acuity-Adaptable Rooms: eliminating the need to transfer patients, improving patient and provider safety, increasing overall satisfaction, and enabling the delivery of higher-quality care.

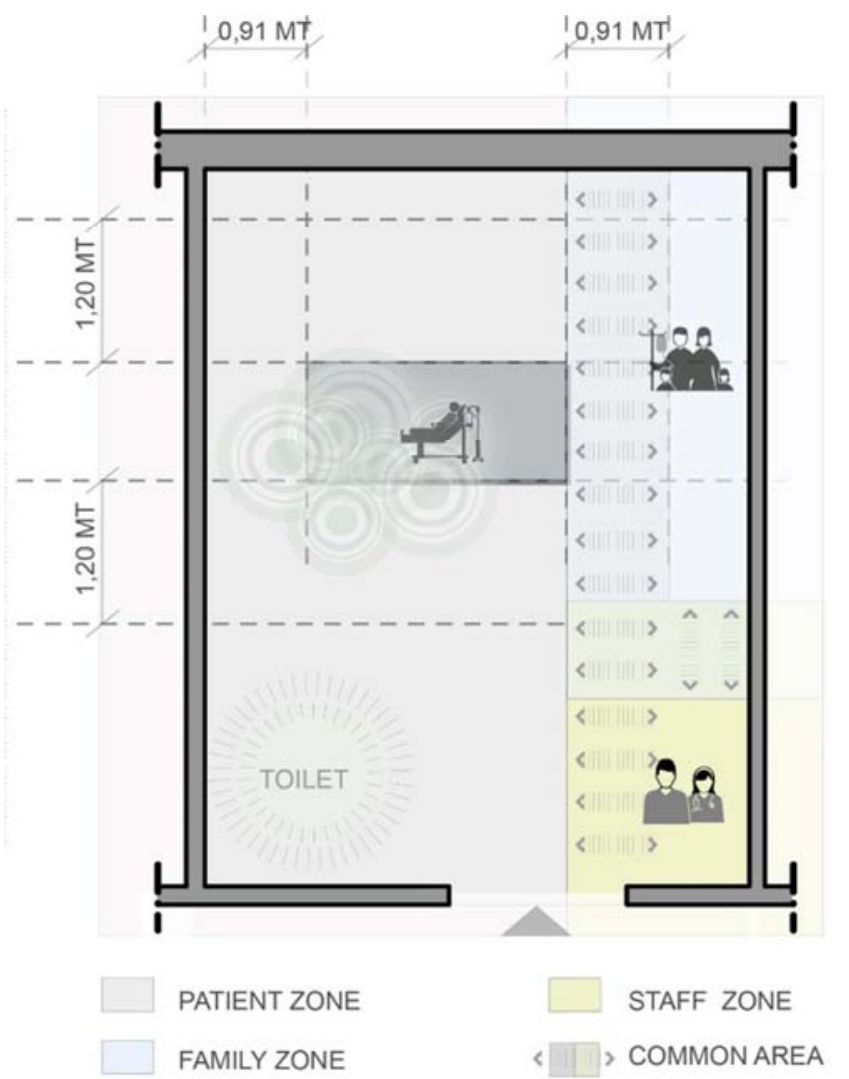

Figure 1. Functional zones.

The formulation of technical data sheets with the design criteria of a patient room model, adaptable to any care intensity represents the final result and the goal of the whole research, whose ultimate aim is to bring innovation in the traditional organization of patient rooms.

\section{Materials and Methods}

\subsection{Functional Zones}

Research has shown that patient healing is influenced by the design of the physical environment [11] and that inpatient room configuration affects staff productivity levels, which influences the patient's health status.

The principles used in designing of the in-patient room focus on maximizing patient safety and improving healing in an atmosphere of patient-centeredness.

To succeed in today's challenging healthcare environment, the design of hospital rooms must take into account primarily the categories of the occupants of the structure: customers (patients and families), staff and physicians. It means that the design of this area has to be very accurate and consider not only the "technological" aspect of the problem but also, and above all, the "human" aspect, related to the patientcenteredness $[12,18,22]$. Any all the measures that allow the patient to spend comfortably his stay in the structure should be consider fundamental.

In particular, the users are: Patient, Caregivers and Family Members.

\subsection{Framework of Needs, Requirements and Criteria}

The framework of needs refers to the users' psychoemotional wellbeing, safety, and the rooms' accessibility. As far as caregivers are concerned, work wellbeing should also be considered.

The in-patient room layout is important because it is easier to create and maintain a reliable culture of safety and patientand-family centered care

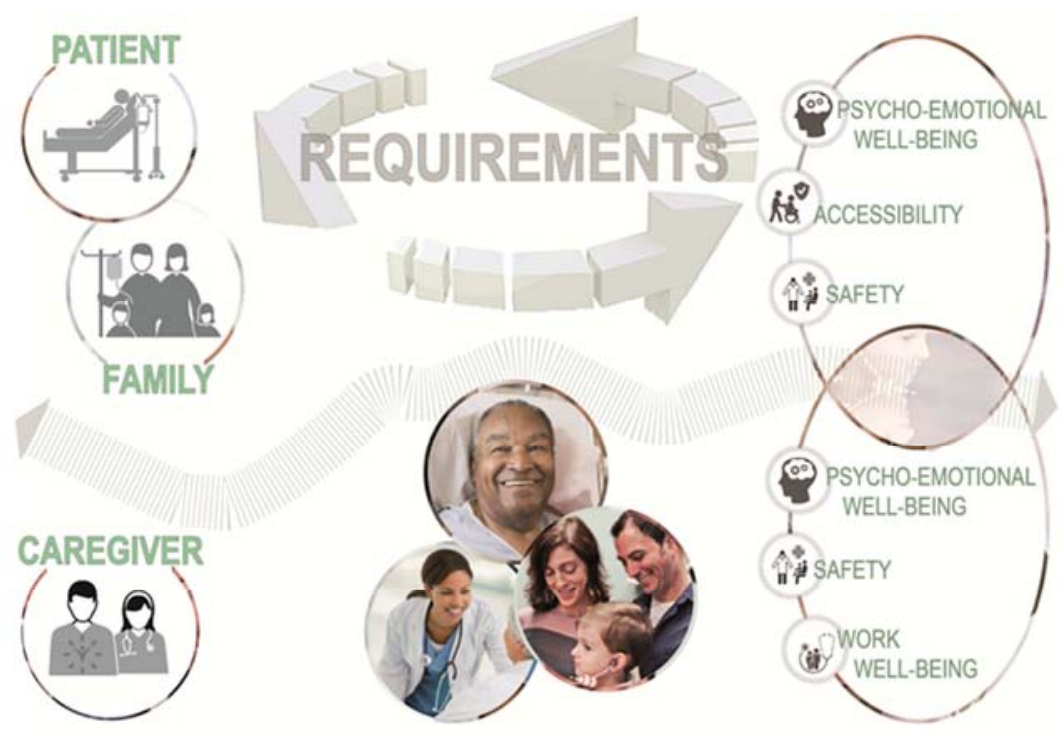

Figure 2. Framework of needs. 
This layout has been designed based on criteria that focus on how the configuration of the in-patient room allows:

a) patients to see their surroundings more easily, so they report less pain and disruption;

b) patients to have easy and safe access to the bathroom, so they are less likely to fall;

c) providers to work more easily, and engage patients and families;

d) providers to maintain eye contact with patients from the hallway, so they can be better aware of the situation of the patient;

e) family members to engage with staff and patients and to comfortably remain in rooms to participate in care.

Table 1. Requirements and design criteria based on the users.

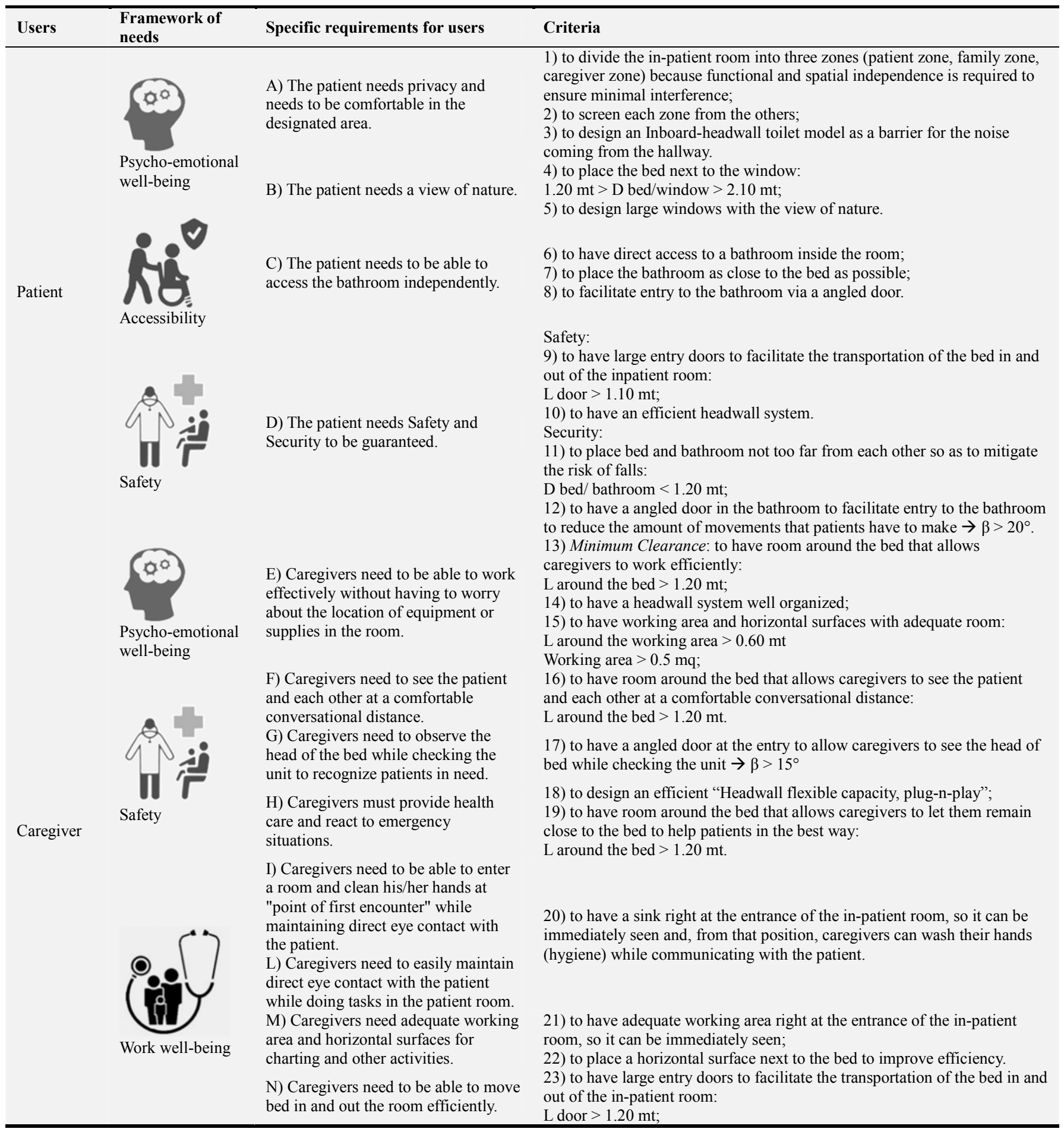




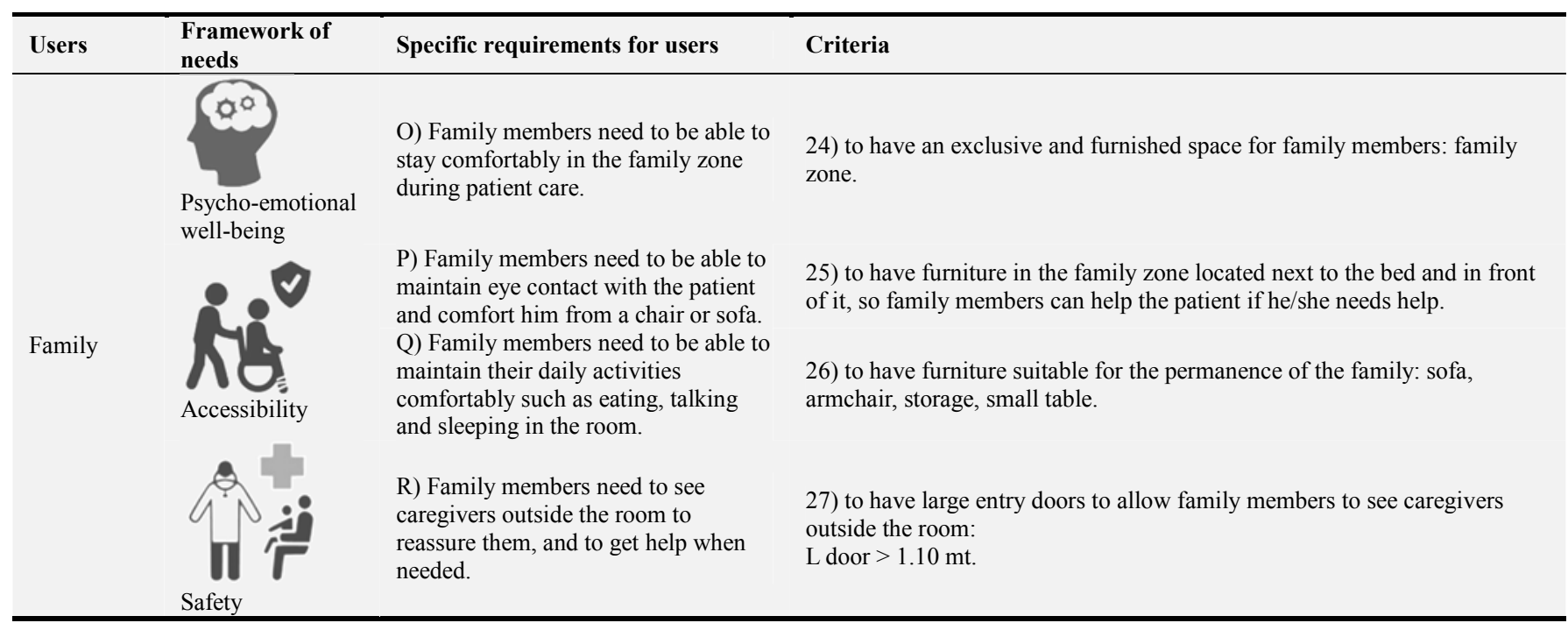

\section{Results and Discussion}

\subsection{Layout of In-patient Rooms}

Individual design features do not always ensure positive results for all related criteria. It is important to think through combinations of design features and the possible positive results.
The key points of the layout of an in-patient room are focused on:

A) "point of first encounter";

B) inboard-headwall bathroom model to optimize accessibility and user safety;

C) headwall, designed to minimize the visual impact of medical devices;

D) footwall, to emphasize the patient-centered care.

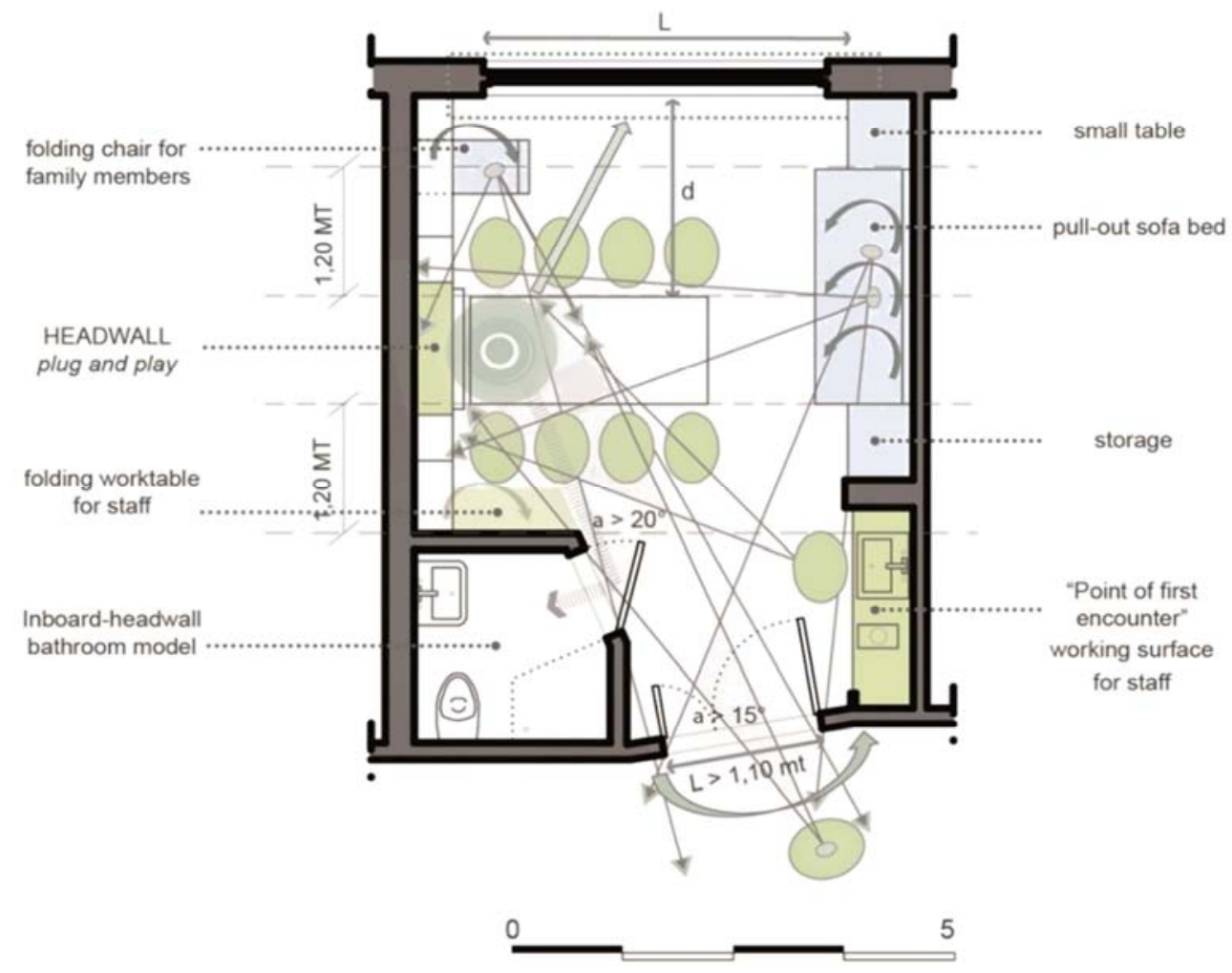

Figure 3. In-patient room layout.

\section{2. "Point of First Encounter"}

The "point of first encounter" is the entrance area to the inpatient room and it has to be designed in relation to the needs related to the patient's privacy and to the possibility of visual control by the staff.

In this area there is an adequate caregivers' working area with a sink located at the immediate entry to the in-patient room, so it can be immediately be seen. From that position, caregivers can wash their hands (hygiene) while 
communicating with the patient. Intended primarily for staff use, it features elements that are designed for sanitation and safety control. In fact, there are compartments to keep disposable items (gowns, masks, headgear, gloves), necessary in case of particularly high levels of asepsis, and a large mirror that allows staff to see the patient while they are washing their hands.

\subsection{Inboard-Headwall Bathroom Model}

The position of the bathroom in the in-patient room is the aspect that has the greatest influence on the overall layout of the in-patient unit in terms of installations. It must be designed to optimize patient usability and staff efficiency. In
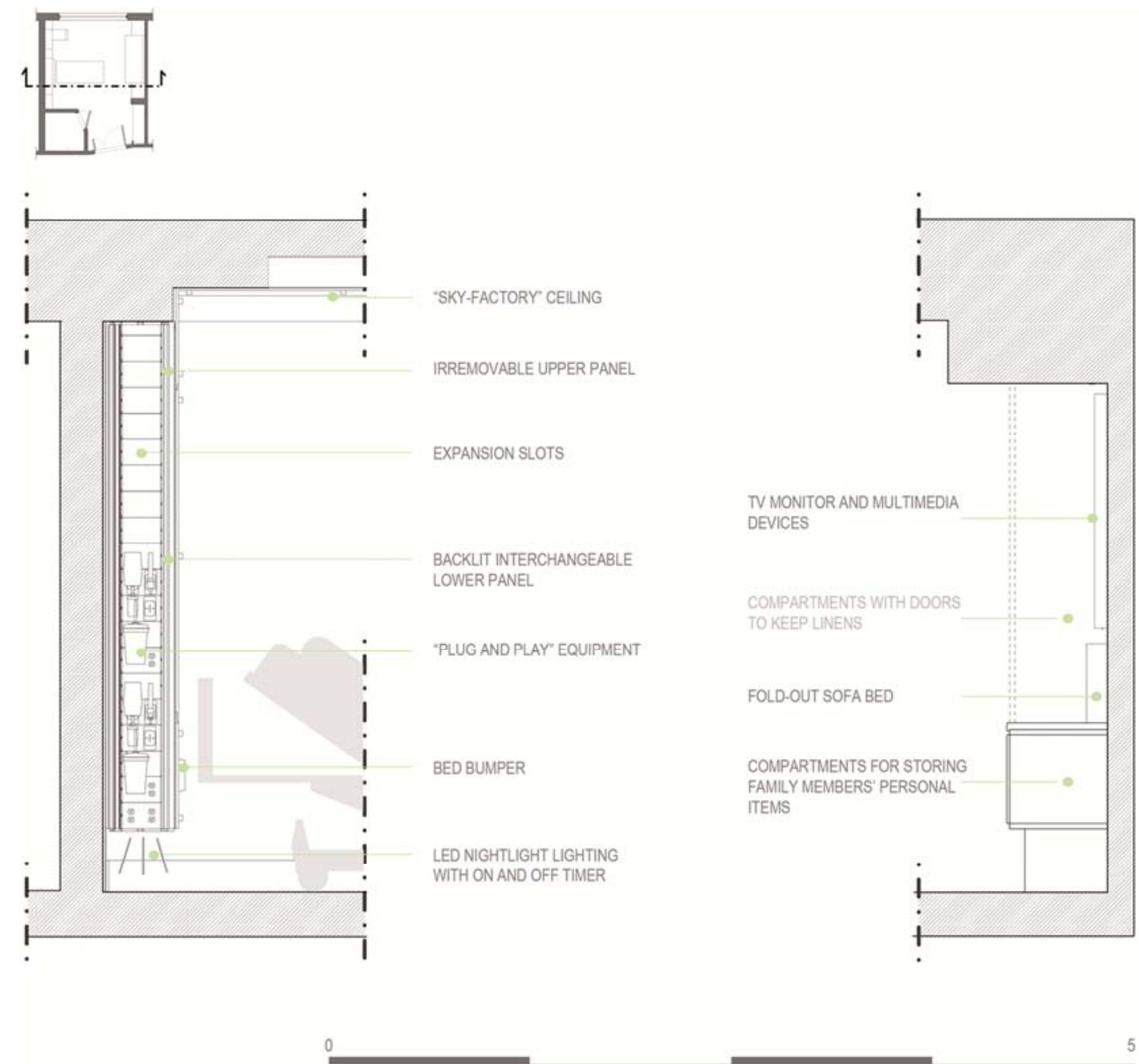

Figure 4. Longitudinal section of in-patient room.

\subsection{Headwall}

The headwall is considered the pivotal point of the project because its design has a direct impact on the experience of patients, family members, and the medical staff.

It provides functions related to the patient and staff and is designed with technological equipment that contains all the additions necessary for the performance of health and hotel activities. It contains the main functional compartments for storing patients' and family members' personal effects and fact, the bathroom is often connected to patients' falls, so the access to the bathroom has important implications for patient safety.

The research proves that the most convenient position of the bathroom is the inboard-headwall position because of its many compelling advantages [13]:

1. Maximum visibility of the patient to the outside (possibility to have big windows), resulting in greater sun-light;

2. Additional space for family members;

3. Privacy barrier to the noise coming from the hallway;

4. Easy accessibility for caregivers. 
"Flexible capacity plug-n-play" [20]. According to the intensity of care, the in-patient room may need different supports and, with the "plug and play" system, the panels can be removed and the interior replaced to adapt to changing functions and the addition of new technologies. It is envisioned as a plug and play kit of parts where the functional elements can be reconfigured and the visual appearance can be tailored to the specific context of the hospital. Flexibility in design is necessary not only to accommodate rapid technology changes, but also to provide services to a continually changing patient population [21].

Easy access and ergonomics are main points of the headwall which contains compartments for the exclusive use of the patient and for family members, and equipment to support the staff located on the two sides of the patient's bed.

\subsection{Footwall}

The footwall is the primary focal point of view for the patient and it is a space usable by the family, which provides a highly accessible and visible staging area for staff as they move in and out of the room.

On the footwall various wall panels are set and provide

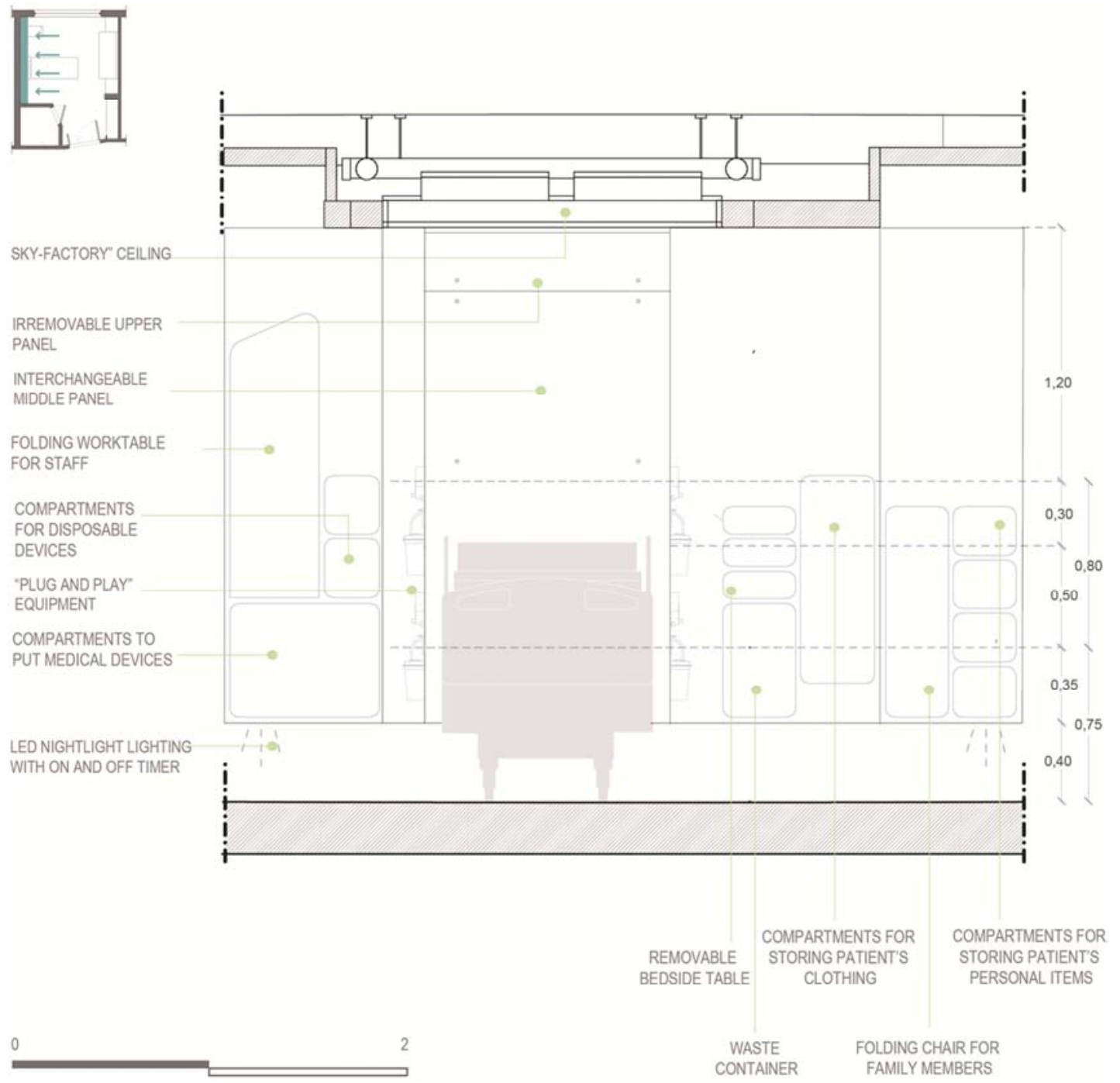

Figure 5. Transversal section of in-patient room opportunities for adaptability and personalization.

There are compartments for clothing and objects belonging to family members and digital devices for entertainment and information (TV with internet access) and a pull-out sofa bed. The arrangement of additional functional components, such as drawers and multiple doors for the storage of personal items and equipment to support the family members, or to keep additional linens (blankets and pillows), underlines the importance of family-centered care and establishes a close relationship between family members and patient.

All functional components do not have handles and are equipped with push-up opening systems in order to facilitate patient opening and minimize dust accumulation surfaces.

Together, the headwall and footwall are important features that directly impact the experience of patients, family, and staff. They represent the opportunity to bring greater innovation and personalization into the typical patient room, and their elements can be adapted or interchanged over the life of the room, or used in various ways during the stay of a patient. 
Finally, another important parameter in defining the room configuration is the Sky Factory system: the ceiling creates photographic illusions with views of the real sky and helps the patient's psycho-emotional well-being. The folds of the false ceiling are also designed to optimize the functionality of the lighting system and to not produce glare phenomena.

\section{Conclusions}

The speed of technology evolution, which makes a hospital obsolete after 10 years long before its physical life is spent, makes necessary new, strong change in health system and the hospital.

The research, through several steps, allows us to understand how to create a latest generation room that is adaptable to sudden changes in the health system.

The processes of change in hospital structures necessarily need strategic management in order to be controlled, oriented and applied.

The formulation of design guidelines through technical data sheets for a in-patient room model represents the starting point for designing a highly innovative health facility that can meet the needs of individual users by adapting to the rapid changes taking place in the healthcare system.

By using these design criteria and incorporating evidencebased concepts, such as the acuity adaptable care delivery model, the new model proposed of in-patient rooms will bring innovation in the traditional organization of patient rooms. It anticipates future needs, and allows for any changes that depend on the various hospital needs, minimizing their costs.

The basic features of a conceptual design of an in-patient room of the future discussed in the article incorporate into the design many recent technological innovations that would allow adaptation and design variations for both function and appearance in each application initially and over time. In fact, the design contains flexible systems that enable adjustments to varying circumstances, alleviating the effects of unpredictable change, and helping healthcare organizations cope better during times of uncertainty.

The use of this system introduces a fundamental change in the design and construction of the in-patient area, inducing the latter to be ready to change over time because of its features which allow, starting from a minimum configuration, to make subsequent upgrades and adaptations to any level of care.

So, the in-patient room model studied brings innovation to the traditional organization of patient rooms that makes it possible to succeed in today's challenging healthcare environment, maximizing patient safety and improving healing; but it must be considered that the complete application of this model in the national health system requires a substantial reorganization of the general hospital services.

The research mentioned that, due to a process of miniaturization of the components and to the possibility of easy transfer of some medical technologies, it is possible to bring these technologies directly to the patient's bed. In this way, the inpatient room can become a real operating room and the formulation of design guidelines through technical data sheets for a in-patient room model has allowed to better establish the efficacy of new concepts before investing in operational environments.

Therfore, a logical next step in future research would be to determine an inpatient room design that is directly associated with operating rooms features in terms of materials and equipments and, if successful, the integration of research into practice stands to change the design process as it exists today.

\section{References}

[1] Mauri M., "The future of the hospital and of the structures of the NHS", Technè 09/2015 (in Italian).

[2] NHS ESTATES, "Ward layouts with single rooms and space for flexibility". Discussion Document- efm-evidence, 2006, pp. 31-45.

[3] Facility Guidelines Institute for Design and Construction of Hospitals and Outpatient Facilities, 2014.

[4] Nickl-Weller C. Nickl H., "Hospital Architecture", Brown, London, 2007.

[5] Atkinson J. Hohenstein J. McCu C., "Using evidence-based strategies to design safe, efficient, and adaptable patient rooms", in Healthcare Design 11 (5), May 2011), pp. 47-54.

[6] Bonuel N. Degracia A. Cesario S., "Acuity-Adaptable Patient Room: Improves Length of Stay and Cost of Patients Undergoing Renal Transplant A Pilot Study", in Critical Care Nursing Quarterly 36 (2), pp. 181-194, April-June 2013.

[7] Lipschutz L. D., "Acuity-adaptable rooms: Design considerations can improve patient care", in Healthcare Construction \& Operations News 7 (1), 2009.

[8] Hendrich A. Chow M., "Maximizing the impact of nursing care quality", in Healthcare Leadership White Paper Series (4 of 5), The Center for Health Design, 2008.

[9] Sadler B. Dubose J. Malone E. Zimring C., "The business case for building better hospitals through evidence based design", in Healthcare Leadership White Paper Series (1 of 5), The Center for Health Design, 2008.

[10] Ulrich R. Zimring C. Zhu X. DuBose J. Seo H. B. Choi Y. S. Quan X. Joseph A., "A Review of the Research Literature on Evidence-Based Healthcare Design", in Healthcare Leadership, white paper series 5 of 5, September 2008.

[11] Glind I. Roode S. Goossensen A., "Do patients in hospitals benefit from single rooms? A literature review", in Health Policy 84 (2-3), December 2007, pp. 153-61.

[12] Capolongo S. Buffoli M., "Quality and physical hospital environment", in Capolongo S., Hospital buildings Methodological and design approaches, Hoepli, Milan, 2006 (in Italian).

[13] Maze C., "Inboard, Outboard, or Nested?", in Healthcare Design 9 (3), May 2009, pp. 38-42. 
[14] Del Nord R. Peretti G., "L'umanizzazione degli spazi di cura", Ministero della Salute, TESIS, 2012.

[15] Abati R. Salerno P., "CORE. La bella utopia. Il grande sogno del Centro oncoematologico di Reggio Emilia diventato realtà", Compagnia Editoriale Aliberti, 2016.

[16] Arduini R., "Innovazione culturale e gestionale in sanità", Franco Angeli, 2016.

[17] Del Nord R., "Le nuove dimensioni strategiche dell'ospedale di eccellenza", Polistampa, 2012.

[18] Giroldi S, Lega F., "L'ospedale modern mette il paziente al centro dell'organizzazione", in Progettare per la Sanità, 2018, pp. 22-27.
[19] Buffoli M. Nachiero D. Capolongo S., "Flexible healthcare structures: analysis and evaluation of possible strategies and technologies", in Ann Ig, 2012.

[20] Brown K. K. Gallant D., "Impacting Patient Outcomes Through Design: Acuity Adaptable Care/Universal Room Design", in Critical Care Nursing Quarterly 29 (4), 2006, pp. 326-341.

[21] Burmahl B., "Facilities of the future. Health Facilities Management”, 13 (2), 2000, pp. 30-34.

[22] Carera S., "A tutto Corian per il benessere del paziente", in Progettare per la Sanità, 2018, pp. 12-16. 